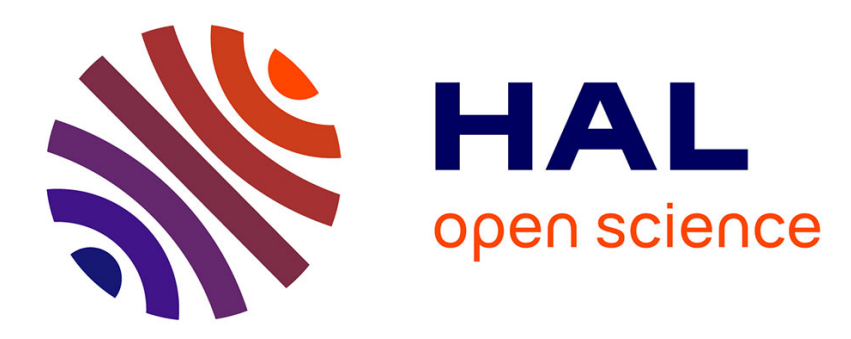

\title{
Patterns in discretized parabolas and length estimation (extended version)
}

Alain Daurat, Mohamed Tajine, Mahdi Zouaoui

\section{To cite this version:}

Alain Daurat, Mohamed Tajine, Mahdi Zouaoui. Patterns in discretized parabolas and length estimation (extended version). DGCI 2009, Sep 2009, Montréal, Canada. pp.737-384, 10.1007/978-3-64204397-0 . hal-00374372v5

\section{HAL Id: hal-00374372 \\ https://hal.science/hal-00374372v5}

Submitted on 6 May 2010

HAL is a multi-disciplinary open access archive for the deposit and dissemination of scientific research documents, whether they are published or not. The documents may come from teaching and research institutions in France or abroad, or from public or private research centers.
L'archive ouverte pluridisciplinaire HAL, est destinée au dépôt et à la diffusion de documents scientifiques de niveau recherche, publiés ou non, émanant des établissements d'enseignement et de recherche français ou étrangers, des laboratoires publics ou privés. 


\title{
Patterns in discretized parabolas and length estimation
}

\author{
(extended version)
}

\author{
Alain Daurat, Mohamed Tajine, and Mahdi Zouaoui \\ LSIIT CNRS UMR 7005, Université de Strasbourg, \\ Pôle API, Boulevard Sébastien Brant, 67400 Illkirch-Graffenstaden, France \\ \{daurat, tajine, mzouaoui\}@unistra.fr
}

\begin{abstract}
We estimate the frequency of the patterns in the discretization of parabolas, when the resolution tends to zero. We deduce that local estimators of length almost never converge to the length for the parabolas.
\end{abstract}

Keywords: Digital Curve, Pattern, Multigrid Convergence.

\section{Introduction}

Length estimation is an important domain of Image Analysis (see [1] for a review). In this paper, we will consider the problem of estimating the length of a curve from its discretizations at different resolutions. In particular we are interested in the comportment of estimators when the resolution tends to zero. We also restrict our study to special estimators called "local estimators" which consist in considering patterns which are pieces of fixed length of the discretized curve. Local estimators simply consist to fix a weight to each pattern and summing these weights to obtain the estimation of length (See Fig. 4 for illustration). So, if we want to study the estimated length by local estimators when the resolution tends to zero, we have at first to study the number of occurrences of a pattern of the discretization of digital curves. In fact an asymptotic result about the occurrence number of patterns for discretized general curves looks to be a quite hard problem, because the discretization process is not a continuous process (the integer part function is not continuous), so the estimation of the occurrence number of patterns cannot be deduced from Mathematical Analysis arguments, but by Number Theory arguments. The two first authors of this paper have already made this study for segments in [2]. In this paper we continue this work by considering another class of curves, the parabolas.

The paper is organized as follows: Section 2 describes the notations used in this paper, Section 3 will be devoted to the study of the frequency of patterns in parabolas, and finally Section 1 will apply the results of this study to the local estimators of length of parabolas. Appendix A contains the detailed proofs which are not in the main part of this paper. 


\section{Notations}

In this section we precise the notations that will be used in all the paper.

- For $x \in \mathbb{R}$ we denote by $\lfloor x\rfloor$ (resp. $\lceil x\rceil$ ) the integer $k$ such that $k \leq x<k+1$ (resp. $k-1<x \leq k$ ).

- The fractional part of $x$ is denoted $\langle x\rangle$ and is defined by $x=\lfloor x\rfloor+\langle x\rangle$.

- For $A, B \in \mathbb{Z}$, the discrete interval $\{A, A+1, \ldots, B-1, B\}$ is denoted $\llbracket A, B \rrbracket$.

- Let $m$ be a positive integer. A pattern of size $m$ is a function $\omega$ from $\llbracket 0, m \rrbracket$ to $\mathbb{Z}$ such that $\omega(0)=0$ and $\omega(k+1) \in\{\omega(k), \omega(k)+1\}$ (see Fig. 11). The set of patterns of size $m$ is denoted $\mathcal{P}_{m}$.

- If $X$ and $Y$ are two real numbers such that $Y>0$ then $X \bmod Y$ is the real number such that $0 \leq X \bmod Y<Y$ and $\frac{X-X \bmod Y}{Y} \in \mathbb{Z}$.

- For $r \in \mathbb{R}$ and $E \subset \mathbb{R}^{2}, r E=\{(r x, r y) \mid(x, y) \in E\}$.

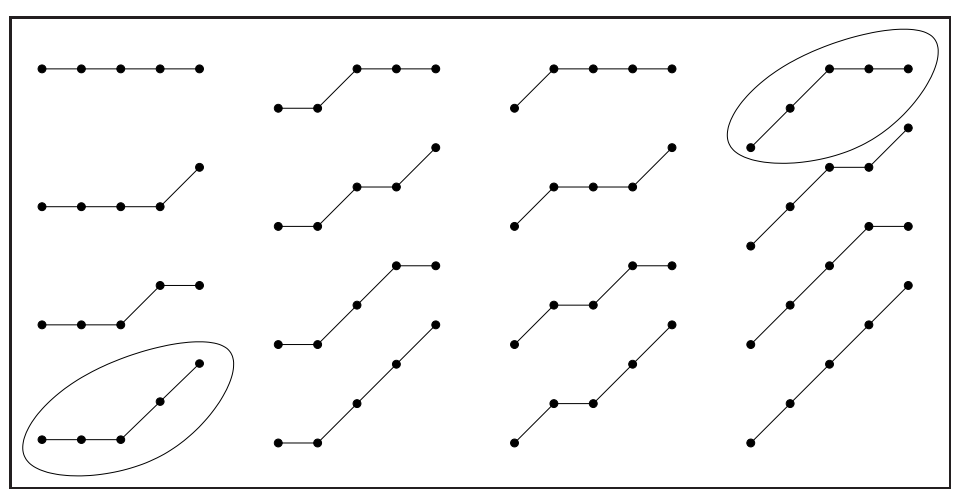

Fig. 1. The 16 patterns of size $m=4$. Only the encircled one are not digital segments.

\section{Frequency of patterns in Discrete Parabolas}

Let $a, b \in \mathbb{R}$ such that $a<b$ and a derivable function $g:[a, b] \rightarrow \mathbb{R}$ which satisfies $0 \leq g^{\prime}(x) \leq 1$ for all $x \in[a, b]$.

In all the following, for any $r>0$ we use the notations:

- $A_{r}=\left\lceil\frac{a}{r}\right\rceil, B_{r}=\left\lfloor\frac{b}{r}\right\rfloor, N_{r}=B_{r}-A_{r}+1$,

- $\mathcal{C}_{r}^{g}=r\left\{(X, Y) \in \mathbb{Z}^{2} \mid A_{r} \leq X \leq B_{r}\right.$ and $\left.Y=\left\lfloor\frac{g(r X)}{r}\right\rfloor\right\}$.

The set $\mathcal{C}_{r}^{g}$ is the "naive" discretization of the graph of $g$ at resolution $r$, and $N_{r}$ is its number of points. 
Let $m$ be a positive integer. The pattern at position $X \in \llbracket A_{r}, B_{r}-m \rrbracket$ of size $m$ of $\mathcal{C}_{r}^{g}$, denoted $\omega_{X, r, m}^{g}$ is defined by:

$$
\omega_{X, r, m}^{g}(k)=\left\lfloor\frac{g(r(X+k))}{r}\right\rfloor-\left\lfloor\frac{g(r X)}{r}\right\rfloor .
$$

The frequency of a pattern $\omega$ of size $m$ in $\mathcal{C}_{r}^{g}$ is defined by:

$$
F_{r}^{g}(\omega)=\frac{\operatorname{card}\left\{X \in \llbracket A_{r}, B_{r}-m \rrbracket \mid \omega_{X, r, m}^{g}=\omega\right\}}{N_{r}-m} .
$$

The aim of this section is the study of $F_{r}^{g}(\omega)$ for some functions $g$. For this we will approximate the curve by its tangents which will also be discretized, so we need some notions about digital straight lines:

For $u \in[0,1]$ and $v \in[0,1)$, let us denote $s_{m}^{u, v}$ the pattern of size $m$ defined by:

$$
s_{m}^{u, v}(k)=\lfloor u k+v\rfloor .
$$

A pattern of this form is called a digital segment of size $m$.

For any pattern $\omega$,

$$
\begin{aligned}
& P I(\omega)=\left\{(u, v) \in[0,1]^{2} \mid\lfloor u k+v\rfloor=\omega(k) \text { for any } k \in \llbracket 0, m \rrbracket\right\}, \\
& \operatorname{pinf}_{u}(\omega)=\inf \{v \mid(u, v) \in P I(\omega)\}, \\
& \operatorname{psup}_{u}(\omega)=\sup \{v \mid(u, v) \in P I(\omega)\} \text {, } \\
& F L_{u}(\omega)=0 \quad \text { if }\{v \mid(u, v) \in P I(\omega)\}=\emptyset \\
& =\operatorname{psup}_{u}(\omega)-\operatorname{pinf}_{u}(\omega) \text { otherwise. }
\end{aligned}
$$

$P I(\omega)$ is called the preimage of $\omega$, it is nonempty if and only if $\omega$ is a digital segment. $F L_{u}(\omega)$ is intuitively the frequency of the pattern $\omega$ in the discretized straight lines of slope $u$. (See [2]3] for more details and [4] for the generalization to slopes of planes).

In all the paper, the considered curves are parabolas corresponding to the function $g(x)=\alpha x^{2}$. We distinguish two cases: the case $\alpha$ irrational and the case $\alpha$ rational. In Subsection 3.1, we will see that for $\alpha$ irrational, the frequency $F_{r}^{g}(\omega)$ converges, when $r$ is rational and tends to zero, to a quantity which can be expressed by using the function $x \mapsto F L_{g^{\prime}(x)}(\omega)$. In Subsection 3.2, we study the case $\alpha$ rational, but we do not succeed to prove a similar result as in the case $\alpha$ irrational. Nevertheless we obtain a weaker result (The Tangent Lemma).

\subsection{Parabolas of equation $y=\alpha x^{2}$ with $\alpha$ irrational}

In this subsection we consider curves $\mathcal{C}_{r}^{g}$ for $g$ defined on $[a, b]$ by $g(x)=\alpha x^{2}$ with $\alpha$ irrational, and $0 \leq a<b \leq \frac{1}{2 \alpha}$. This last hypothesis is needed to have $0 \leq g^{\prime}(x) \leq 1$ for $x \in[a, b]$.

The main result of this subsection is the following: 
Theorem 1. If $g(x)=\alpha x^{2}$ with $\alpha \notin \mathbb{Q}$ then for any pattern $\omega$ we have

$$
F_{r}^{g}(\omega) \underset{\substack{r \rightarrow 0 \\ r \in \mathbb{Q}}}{\longrightarrow} \frac{1}{b-a} \int_{a}^{b} F L_{g^{\prime}(x)}(\omega) \mathrm{d} x
$$

The rest of this subsection is devoted to the proof of this Theorem.

The first needed lemma shows that the discretization of a curve and the discretization of its tangent are similar near the origin of the tangent and when the resolution tends to zero:

Lemma 1 (Tangent Lemma). If $g$ is defined on $[a, b]$ by $g(x)=\alpha x^{2}$ with $\alpha$ irrational, and $0 \leq a<b \leq \frac{1}{2 \alpha}$ then

$$
\frac{\operatorname{card}\left\{X \in \llbracket A_{r}, B_{r}-m \rrbracket \mid \omega_{X, r, m}^{g} \neq s_{m}^{g^{\prime}(r X),\left\langle\frac{g(r X)}{r}\right\rangle}\right\}}{N_{r}-m} \underset{\substack{r \rightarrow 0 \\ r \in \mathbb{Q}}}{\longrightarrow} 0 .
$$

Lemma $\mathbb{1}$ is illustrated by Fig. 2. In the last lemma we consider $r \in \mathbb{Q}$ because its proof needs the irrationality of $\alpha r$.

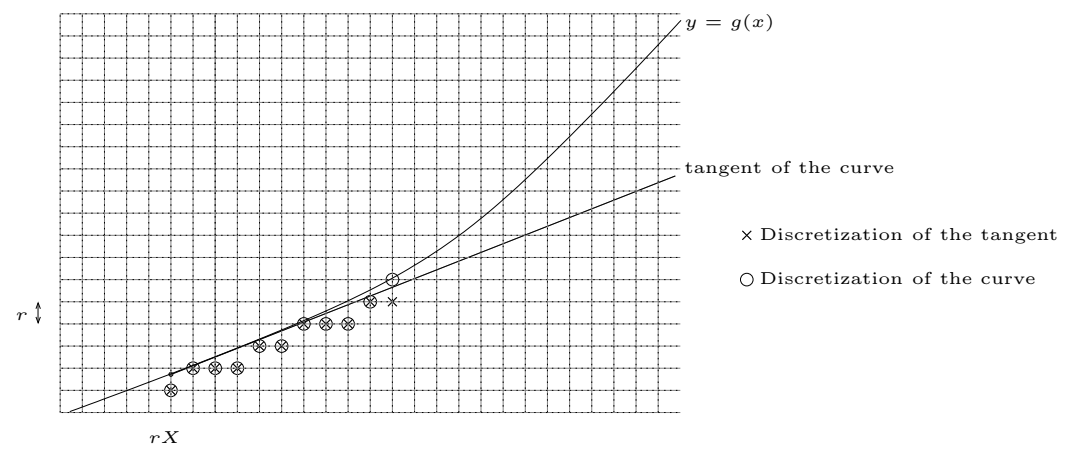

Fig. 2. Comparison of the discretization of the curve and the discretization of its tangent : here we have $\omega_{X, r, m}^{g}=s_{m}^{g^{\prime}(r X),\left\langle\frac{g(r X)}{r}\right\rangle}$ for $m=9$ but not for $m=10$.

Before starting the proof of Lemma 1 we need one more notation and two other lemmas. For $X \in \llbracket A_{r}, B_{r} \rrbracket$, we define $P_{r, k}(X)$ by:

$$
P_{r, k}(X)=\frac{g(r X)}{r}+g^{\prime}(r X) k=\alpha r X^{2}+2 \alpha k r X=\alpha r\left((X+k)^{2}-k^{2}\right) .
$$

This definition is motivated by the following lemma:

Lemma 2. If $r<\frac{1}{\alpha m^{2}}$, then for any $X$ we have $\omega_{X, r, m}^{g}=s_{m}^{g^{\prime}(r X),\left\langle\frac{g(r X)}{r}\right\rangle}$ if and only if for all $k \in \llbracket 0, m \rrbracket$ we have $\left\langle P_{r, k}(X)\right\rangle<1-\alpha r k^{2}$. 
Proof.

$$
\begin{aligned}
\omega_{X, r, m}^{g}(k) & =\left\lfloor\frac{g(r(X+k))}{r}\right\rfloor-\left\lfloor\frac{g(r X)}{r}\right\rfloor \\
& =\left\lfloor\frac{g(r X)}{r}+g^{\prime}(r X) k+\alpha r k^{2}\right\rfloor-\left\lfloor\frac{g(r X)}{r}\right\rfloor
\end{aligned}
$$

We know that $\lfloor u+v\rfloor=\lfloor u\rfloor+\lfloor v\rfloor$ iff $\langle u\rangle+\langle v\rangle<1$.

So $\omega_{X, r, m}^{g}(k)=\left\lfloor\frac{g(r X)}{r}+g^{\prime}(r X) k\right\rfloor-\left\lfloor\frac{g(r X)}{r}\right\rfloor$ iff $\left\langle\frac{g(r X)}{r}+g^{\prime}(r X) k\right\rangle+\left\langle\alpha r k^{2}\right\rangle<1$.

With the hypothesis $r<\frac{1}{\alpha m^{2}}$ we have $\left\langle\alpha r k^{2}\right\rangle=\alpha r k^{2}$. But:

$$
\begin{gathered}
\left\lfloor\frac{g(r X)}{r}+g^{\prime}(r X) k\right\rfloor-\left\lfloor\frac{g(r X)}{r}\right\rfloor=\left\lfloor\left\langle\frac{g(r X)}{r}\right\rangle+g^{\prime}(r X) k\right\rfloor-\left\lfloor\left\langle\frac{g(r X)}{r}\right\rangle\right\rfloor=s_{m}^{g^{\prime}(r X),\left\langle\frac{g(r X)}{r}\right\rangle}(k) \\
\text { So } \omega_{X, r, m}^{g}=s_{m}^{g^{\prime}(r X),\left\langle\frac{g(r X)}{r}\right\rangle} \text { iff for all } k \in \llbracket 0, m \rrbracket \text { we have }\left\langle P_{r, k}(X)\right\rangle<1-\alpha r k^{2} .
\end{gathered}
$$

Lemma 3. Let I be an interval of $[0,1]$. We have $T_{r, k}(I) \underset{\substack{r \rightarrow 0 \\ r \in \mathbb{Q}}}{\longrightarrow} \mu(I)$ where

$$
T_{r, k}(I)=\frac{1}{N_{r}} \operatorname{card}\left\{X \in \llbracket A_{r}, B_{r} \rrbracket \mid\left\langle P_{r, k}(X)\right\rangle \in I\right\}
$$

and $\mu(I)$ is the usual length of $I$.

The proof of this lemma uses Weyl's argument, as in the proof of Theorem 1 of [2] or Appendix A.1 of [4, but extended to the quadratic case following the same ideas as in [5, p6-7]. It is given in Appendix A.1.

Proof of Lemma 耳. We recall that $\omega_{X, r, m}^{g}=s_{m}^{g^{\prime}(r X),\left\langle\frac{g(r X)}{r}\right\rangle}$ iff for all $k \in \llbracket 0, m \rrbracket$ we have $\left\langle P_{r, k}(X)\right\rangle<1-\alpha r k^{2}$ so:

$$
\frac{\operatorname{card}\left\{X \in \llbracket A_{r}, B_{r}-m \rrbracket \mid \omega_{X, r, m}^{g} \neq s_{m}^{g^{\prime}(r X),\left\{\frac{g(r X)}{r}\right\rangle}\right\}}{N_{r}-m} \leq \frac{N_{r}}{N_{r}-m} \max _{k=0} T_{r, k}\left(I_{r, k}\right)
$$

where $I_{r, k}=\left[1-\alpha r k^{2}, 1\right)$, so it is sufficient to show that $T_{r, k}\left(I_{r, k}\right) \underset{\substack{r \rightarrow 0 \\ r \in \mathbb{Q}}}{\longrightarrow} 0$ for any $k \in \llbracket 0, m \rrbracket$. Let $\varepsilon>0$, there exists $R_{1}>0$ such that for any $r<R_{1}$ we have $\alpha r k^{2}<\frac{\varepsilon}{2}$. We know by Lemma 3 that there exists $R_{2}>0$ such that for any $r<R_{2}$ we have $T_{r, k}\left(I_{R_{0}, k}\right) \leq \mu\left(I_{R_{0}, k}\right)+\frac{\varepsilon}{2}$. If $r$ is such that $r<\min \left(R_{1}, R_{2}\right)$, we have:

$$
\begin{aligned}
S_{r, k}\left(I_{r, k}\right) & \leq S_{r, k}\left(I_{R_{1}, k}\right) \\
& \leq \mu\left(I_{R_{1}, k}\right)+\frac{\varepsilon}{2} \\
& =\alpha r k^{2}+\frac{\varepsilon}{2} \\
& \leq \frac{\varepsilon}{2}+\frac{\varepsilon}{2}=\varepsilon
\end{aligned}
$$

This finishes the proof of Lemma 1 
Sketch of Proof of Theorem $⿴$. We present here the main ideas of the proof of Theorem 1. The detailed proof is in Appendix A.2.

By using Lemma 1 we know that $F_{r}^{g}(\omega)$ has the same limit as $r$ tends to zero as:

$$
G_{r}^{g}(\omega)=\frac{\operatorname{card}\left\{X \in \llbracket A_{r}, B_{r}-m \rrbracket \mid s_{m}^{g^{\prime}(r X),\left\langle\frac{g(r X)}{r}\right\rangle}=\omega\right\}}{N_{r}-m} .
$$

But $s_{m}^{x, y}=\omega$ is equivalent to $(x,\langle y\rangle) \in P I(\omega)$ so:

$$
G_{r}^{g}(\omega)=\frac{\operatorname{card}\left\{X \in \llbracket A_{r}, B_{r}-m \rrbracket \mid\left(g^{\prime}(r X),\left\langle\frac{g(r X)}{r}\right\rangle\right) \in P I(\omega)\right\}}{N_{r}-m}
$$

which has the same limit as $H_{r}(P I(\omega))$ where

$$
H_{r}(E)=\frac{\operatorname{card}\left\{X \in \llbracket A_{r}, B_{r} \rrbracket \mid\left(g^{\prime}(r X),\left\langle\frac{g(r X)}{r}\right\rangle\right) \in E\right\}}{B_{r}-A_{r}+1} .
$$

By applying Lemma 3 with $k=0$ to the piece of the curve $y=g(x)$ restricted to the domain $g^{\prime-1}\left(\alpha_{1}\right) \leq x \leq g^{\prime-1}\left(\alpha_{2}\right)$, we can prove:

$$
H_{r}\left(\left[\alpha_{1}, \alpha_{2}\right) \times I\right) \underset{\substack{r \rightarrow 0 \\ r \in \mathbb{Q}}}{\longrightarrow} \frac{g^{\prime-1}\left(\alpha_{2}\right)-g^{\prime-1}\left(\alpha_{1}\right)}{b-a} \mu(I)
$$

So by approximating $P I(\omega)$ as the union of rectangles

$$
\bigcup_{i=1}^{n}\left[y_{i-1}, y_{i}\right) \times\left[\operatorname{pinf}_{y_{i}}(\omega), \operatorname{psup}_{y_{i}}(\omega)\right)
$$

we approximate $H_{r}(P I(\omega))$ by:

$$
\sum_{i=1}^{n} \frac{g^{\prime-1}\left(y_{i}\right)-g^{\prime-1}\left(y_{i-1}\right)}{b-a} F L_{y_{i}}(\omega)
$$

which is a Riemann sum for $\int_{a}^{b} F L_{g^{\prime}(x)}(\omega) \mathrm{d} x$.

Corollary 1. If $g(x)=\alpha x^{2}$ with $\alpha \notin \mathbb{Q}$, then for any pattern $\omega$ which is not a digital segment we have

$$
F_{r}^{g}(\omega) \underset{\substack{r \rightarrow 0 \\ r \in \mathbb{Q}}}{\longrightarrow} 0
$$

Numerical Application: We illustrate Theorem 1 with an example. Consider the curve $\mathcal{C}$ defined $y=g(x)=\frac{1}{\sqrt{2}} x^{2}$ for $x$ between $a=0$ and $b=\frac{1}{\sqrt{2}}$, and the pattern $\omega$ of size $m=3$ defined by $(\omega(0), \omega(1), \omega(2), \omega(3))=(0,1,2,2)$. We will compute the limit of the frequency of $\omega$ when the resolution tends to zero. 
First we can compute easily $F L_{\alpha}(\omega)$ because $\alpha \mapsto F L_{\alpha}(\omega)$ is a continuous function which is affine between two $m$-Farey numbers (see [3]). So we deduce:

$$
\begin{aligned}
F L_{\alpha}(\omega) & =0 & & \text { if } \alpha \in\left[0, \frac{1}{2}\right] \\
& =2 \alpha-1 & & \text { if } \alpha \in\left[\frac{1}{2}, \frac{2}{3}\right] \\
& =1-\alpha & & \text { if } \alpha \in\left[\frac{2}{3}, 1\right]
\end{aligned}
$$

Theorem 11 proves that:

$$
\begin{aligned}
F_{r}^{g}(\omega) \underset{\substack{r \rightarrow 0 \\
r \in \mathbb{Q}}}{\longrightarrow} & \frac{1}{b-a} \int_{a}^{b} F L_{g^{\prime}(x)}(\omega) \mathrm{d} x \\
= & \sqrt{2} \int_{0}^{\frac{1}{\sqrt{2}}} F L_{\sqrt{2} x}(\omega) \mathrm{d} x \\
= & \sqrt{2}\left(\int_{\frac{1}{2 \sqrt{2}}}^{\frac{2}{3 \sqrt{2}}}(2 \sqrt{2} x-1) \mathrm{d} x+\int_{\frac{2}{3 \sqrt{2}}}^{\frac{1}{\sqrt{2}}}(1-\sqrt{2} x) \mathrm{d} x\right)=\frac{1}{12}
\end{aligned}
$$

\subsection{Parabolas of equation $y=\alpha x^{2}$ with $\alpha$ rational}

Now we are interested in the case where $\alpha$ is rational. Theorem 1 can be generalized to $\alpha$ rational and to the irrational resolutions $r$ because only the irrationality of $\alpha r$ is used in the proof of this theorem.

On the contrary, in the case $\alpha$ rational and rational resolutions, the only result we will prove in this subsection is about the Tangent Lemma. Moreover, we must impose some restrictions about the resolution $r$ and the interval $[a, b]$ of definition of the parabola: $r \alpha=\frac{1}{p}$ where $p$ is a prime number ; $a=0$ and $b=\frac{1}{2 \alpha}$. In all this subsection we suppose that these conditions are satisfied. Actually, we do not succeed to prove the Tangent Lemma in the general case for $\alpha$ rational.

Let $P_{r, k}(X)=r \alpha\left((X+k)^{2}-k^{2}\right)$. We will prove that for any interval $I \subset[0,1]$

$$
\lim _{\substack{r \rightarrow 0 \\ r \alpha}} \frac{\operatorname{card}\left\{X \in \llbracket A_{r}, B_{r}-k \rrbracket \mid\left\langle P_{r, k}(x)\right\rangle \in I\right\}}{N_{r}-k}=\mu(I)
$$

Definition 1. Let $p$ be a prime number and a be an integer number, we define the Legendre symbol $\left(\frac{a}{p}\right)$ of a relatively to $p$ by

$$
\left(\frac{a}{p}\right)=\left\{\begin{array}{l}
0 \quad \text { if } p \text { divides } a \\
1 \quad \text { if a there exists } t \in \mathbb{Z} \text { such that a } \bmod p=t^{2} \bmod p \\
-1 \text { otherwise }
\end{array}\right.
$$


Property 1. (Pólya-Vinogradov inequality [6], [7, Chap. 23]) Let $p$ be a prime number and $M$ and $N$ two positive integers then

$$
\left|\sum_{n=M}^{M+N}\left(\frac{n}{p}\right)\right|<\sqrt{p} \log (p)
$$

Corollary 2. Let $J=\llbracket M, M+N \rrbracket$ be an integer interval. Then

$$
\left|\frac{\operatorname{card}(J)}{2}-\operatorname{card}\left\{y \in I \mid\left(\frac{y}{p}\right)=1\right\}\right|<\sqrt{p} \log (p) .
$$

Lemma 4. Let $\alpha$ be a rational number and assume that $a=0$ and $b=\frac{1}{2 \alpha}$. Then, for any interval $I \subset[0,1]$

$$
\lim _{r \rightarrow 0} \frac{\operatorname{card}\left\{X \in \llbracket A_{r}, B_{r}-k \rrbracket \mid\left\langle P_{r, k}(X)\right\rangle \in I\right\}}{N_{r}-k}=\mu(I)
$$

where the limit is taken on the $r$ such that $r>0$ and $r \alpha=\frac{1}{p}$ where $p$ is a prime number.

Proof. The function $X \mapsto X^{2} \bmod p$ from $\llbracket 1, \frac{p-1}{2} \rrbracket$ to $\left\{Y \mid\left(\frac{Y}{p}\right)=1\right\}$ is a bijection.

Put $H_{r}=\frac{\operatorname{card}\left\{X \in \llbracket A_{r}, B_{r}-k \rrbracket \mid\left\langle P_{r, k}(x)\right\rangle \in I\right\}}{N_{r}}$. Then

$H_{r}=\frac{\operatorname{card}\left\{X \in \llbracket k,\left\lfloor\frac{p}{2}\right\rfloor \rrbracket \mid\left\langle\frac{X^{2}-k^{2}}{p}\right\rangle \in I\right\}}{N_{r}-k}=\frac{2 \operatorname{card}\left\{X \in \llbracket k, \frac{p-1}{2} \rrbracket \mid \frac{X^{2} \bmod p-k^{2}}{p} \in I\right\}}{p+1-2 k}$

Thus

$\operatorname{card}\left\{X \in \llbracket 1, \frac{p-1}{2} \rrbracket \mid X^{2} \bmod p \in J\right\}=\operatorname{card}\left\{Y \in J \mid\left(\frac{Y}{p}\right)=1\right\}$ where $J=p I+k^{2}$.

So $\left|\operatorname{card}\left\{X \in \llbracket k, \frac{p-1}{2} \rrbracket \mid X^{2} \bmod p \in J\right\}-\operatorname{card}\left\{Y \in J \mid\left(\frac{Y}{p}\right)=1\right\}\right|<k$.

By using Pólya-Vinogradov inequality we have:

$$
\left|\frac{\operatorname{card}(J)}{2}-\operatorname{card}\left\{X \in \llbracket 1, \frac{p-1}{2} \rrbracket \mid X^{2} \bmod p \in J\right\}\right|<\sqrt{p} \log (p)
$$

So, $\left|\frac{\operatorname{card}(J)}{2}-\operatorname{card}\left\{X \in \llbracket k, \frac{p-1}{2} \rrbracket \mid X^{2} \bmod p \in J\right\}\right|<\sqrt{p} \log (p)+k$.

By (4) we have

$\left|\frac{2}{p+1-2 k} \frac{\operatorname{card}(J)}{2}-\frac{2}{p+1-2 k} \operatorname{card}\left\{X \in \llbracket k, \frac{p-1}{2} \rrbracket \mid X^{2} \bmod p \in J\right\}\right|<\frac{2 \sqrt{p} \log (p)+2 k}{p+1-2 k}$

Thus, $\lim _{r \rightarrow 0} \frac{\operatorname{card}\left\{X \in \llbracket A_{r}, B_{r}-k \rrbracket \mid\left\langle P_{r, k}(X)\right\rangle \in I\right\}}{N_{r}-k}=\mu(I)$ 
Theorem 2 (Tangent Lemma for rational slopes and special rational resolutions). For any rational $\alpha>0$ and any $a, b$ such that $0 \leq a<b \leq \frac{1}{2 \alpha}$ we have

$$
\frac{\operatorname{card}\left\{X \in \llbracket A_{r}, B_{r}-m \rrbracket \mid \omega_{X, r, m}^{g} \neq s_{m}^{g^{\prime}(r X),\left\{\frac{g(r X)}{r}\right\rangle}\right\}}{N_{r}-m} \underset{\frac{1}{r \alpha} \text { is prime }}{\stackrel{\substack{r \rightarrow 0 \\ \text { prime }}}{\longrightarrow}} 0
$$

Proof. The case where $a=0, b=\frac{1}{2 \alpha}$ can be proved exactly in the same way as for Lemma 1 by using Lemma 1 instead of Lemma 3 . Consider the general case $[a, b] \subset\left[0, \frac{1}{2 \alpha}\right]$. Then we know that:

$$
\begin{aligned}
& \frac{\operatorname{card}\left\{X \in \llbracket A_{r}, B_{r}-m \rrbracket \mid \omega_{X, r, m}^{g} \neq s_{m}^{g^{\prime}(r X),\left\{\frac{g(r X)}{r}\right\rangle}\right\}}{N_{r}-m} \\
& \quad \leq \frac{\left\lfloor\frac{1}{2 \alpha r}\right\rfloor+1-m}{N_{r}-m} \cdot \frac{\operatorname{card}\left\{X \in \llbracket 0,\left\lfloor\frac{b}{r}\right\rfloor-m \rrbracket \mid \omega_{X, r, m}^{g} \neq s_{m}^{g^{\prime}(r X),\left\{\frac{g(r X)}{r}\right\rangle}\right\}}{\left\lfloor\frac{1}{2 \alpha r}\right\rfloor+1-m} \\
& \quad \underset{\frac{1}{r \alpha} \text { is prime }}{\longrightarrow} \frac{\frac{1}{2 \alpha}}{b-a} \cdot 0=0 \quad \text { because of the case } a=0, b=\frac{1}{2 \alpha} .
\end{aligned}
$$

Unfortunately we do not successfully generalize Theorem 1 to rational $\alpha$ and some rational resolutions even if experimentally Theorem it seems to be true in all the cases.

\section{Application to local estimators of length}

A local estimator is given by a weight function $p$ from the set $\mathcal{P}_{m}$ of patterns of size $m$ to $\mathbb{R}$. The estimated length of the curve $y=g(x)$ where $g:[a, b] \rightarrow \mathbb{R}$ at resolution $r$ is given by:

$$
l(p, g, r)=r \sum_{k=0}^{\left\lfloor\frac{B_{r}-m-A_{r}}{m}\right\rfloor} p\left(\omega_{A_{r}+k m, r, m}^{g}\right) .
$$

Theorem 3. Let $a, b$ such that $0 \leq a<b$. The length estimated by a local estimator of a parabola $y=\alpha x^{2}, x \in[a, b],\left(\alpha \leq \frac{1}{2 b}\right)$ converges when the resolution $r$ is rational and tends to zero, to the length of the parabola only for a finite number of irrational numbers $\alpha$.

Sketch of proof. For this proof we use the notation $[f(x)]_{a}^{b}=f(b)-f(a)$.

It is easy to see that:

$$
l(p, g, r)-\frac{(b-a)}{m} \sum_{\omega \in \mathcal{P}_{m}} p(\omega) F_{r}^{\prime g}(\omega) \underset{r \rightarrow 0}{\longrightarrow} 0
$$




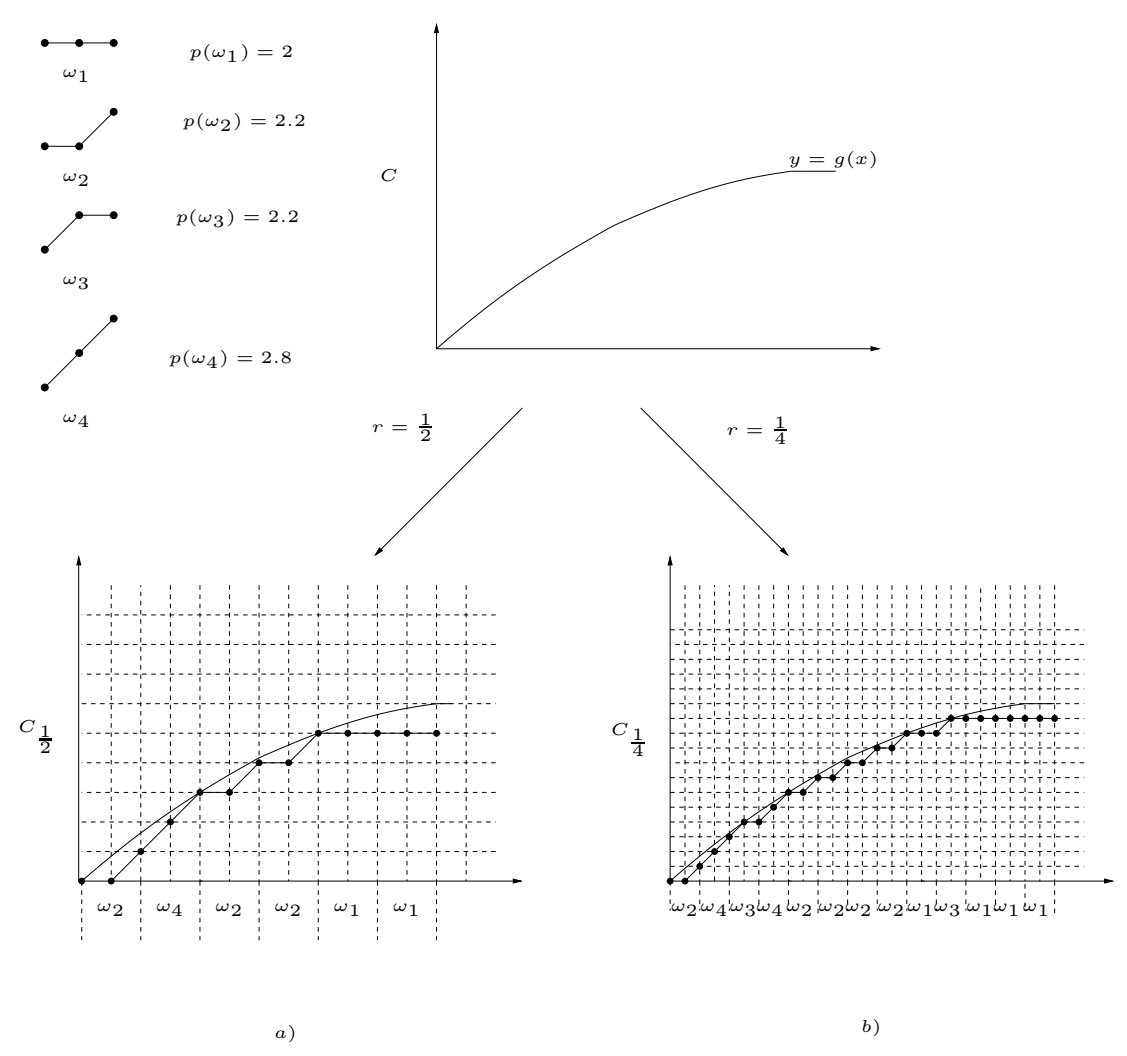

Fig. 3. Estimation of length of a curve from its discretization for two different resolutions: a) $l\left(p, g, \frac{1}{2}\right)=\frac{1}{2}\left(2 p\left(\omega_{1}\right)+3 p\left(\omega_{2}\right)+1 p\left(\omega_{4}\right)\right)=6.7, \quad$ b) $l\left(p, g, \frac{1}{4}\right)=$ $\frac{1}{4}\left(4 p\left(\omega_{1}\right)+5 p\left(\omega_{2}\right)+2 p\left(\omega_{3}\right)+2 p\left(\omega_{4}\right)\right)=7.1$

where

$$
F_{r}^{\prime}(\omega)=\frac{\operatorname{card}\left\{X \in \llbracket A_{r}, B_{r}-m \rrbracket \cap\left(A_{r}+m \mathbb{Z}\right) \mid \omega_{X, r, m}^{g}=\omega\right\}}{\left\lfloor\frac{B_{r}-m-A_{r}}{m}\right\rfloor} .
$$

Consider again the curve defined by $g(x)=\alpha x^{2}$ for $\alpha \notin \mathbb{Q}$. The proof of Theorem 1 can be extended to prove that:

$$
F_{r}^{\prime g}(\omega) \underset{r \in \mathbb{Q}}{\underset{r \rightarrow 0}{\longrightarrow}} \frac{1}{b-a} \int_{a}^{b} F L_{g^{\prime}(x)}(\omega) \mathrm{d} x .
$$

So,

$$
l(p, g, r) \underset{\substack{r \rightarrow 0 \\ r \in \mathbb{Q}}}{\longrightarrow} \frac{1}{m} \sum_{\omega \in \mathcal{P}_{m}} p(\omega) \int_{a}^{b} F L_{g^{\prime}(x)}(\omega) \mathrm{d} x .
$$


We know that $x \mapsto F L_{x}(\omega)$ is piecesewisely affine ([3]). From this property we deduce that we can partition the interval $\left[0, \frac{1}{2 b}\right]$ in a finite number of intervals $\left(I_{k}\right)_{0 \leq k \leq n}$ such that $L_{e s t}(\alpha)=\lim _{r \rightarrow \mathbb{Q}} l(p, g, r)$ is of the form $\frac{A}{\alpha}+B \alpha+C$ on each interval $I_{k}$. (See Appendix A.3 for the details).

Let $L_{\text {real }}(\alpha)$ be the length of the parabola $\left\{\left(x, \alpha x^{2}\right) \mid x \in[a, b]\right\}$. We have:

$$
L_{\text {real }}(\alpha)=\int_{a}^{b} \sqrt{1+(2 \alpha x)^{2}} \mathrm{~d} x=\left[\frac{x \sqrt{1+(2 \alpha x)^{2}}}{2}+\frac{\arg \sinh (2 \alpha x)}{4 \alpha}\right]_{a}^{b}
$$

Suppose that $L_{\text {real }}(\alpha)=L_{\text {est }}(\alpha)$ for an infinite number of irrational numbers $\alpha$, then there exists an interval $I_{k}$ of the previous partition of $\left[0, \frac{1}{2 b}\right]$ such that $L_{\text {real }}(\alpha)=L_{\text {est }}(\alpha)$ for an infinite number of irrational numbers $\alpha \in I_{k}$. On $I_{k}$ we know that $L_{e s t}(\alpha)$ has the form $\frac{A}{\alpha}+B \alpha+C$. The functions $\alpha \mapsto \alpha L_{\text {real }}(\alpha)$ and $\alpha \mapsto \alpha\left(\frac{A}{\alpha}+B \alpha+C\right)$ are holomorphic in a open set of $\mathbb{C}$ containing $\left[0, \frac{1}{2 b}\right]$ and are equal for an infinite number of $\alpha \in I_{k} \subset\left[0, \frac{1}{2 b}\right]$. So by Theorem on the zeros of holomorphic functions [8, Cha. 10] they are equal on $\left[0, \frac{1}{2 b}\right]$. So:

$$
\alpha L_{\text {real }}(\alpha)=A+B \alpha^{2}+C \alpha \quad \text { for all } \alpha \in\left[0, \frac{1}{2 b}\right]
$$

We have:

$$
\begin{aligned}
\frac{\partial\left(\alpha L_{\text {real }}(\alpha)\right.}{\partial \alpha} & =b \sqrt{1+(2 \alpha b)^{2}}-a \sqrt{1+(2 \alpha a)^{2}} \\
& =b-a+2\left(b^{3}-a^{3}\right) \alpha^{2}+o\left(\alpha^{2}\right) \quad \text { when } \alpha \rightarrow 0
\end{aligned}
$$

But $\frac{\partial\left(A+B \alpha^{2}+C \alpha\right)}{\partial \alpha}=2 B \alpha+C$, so $2\left(b^{3}-a^{3}\right)=0$ which is impossible if $b>a$. So the hypothesis that $L_{\text {real }}(\alpha)=L_{\text {est }}(\alpha)$ for an infinite number of irrational $\alpha$ is absurd.

Corollary 3. Let $a, b$ such that $0 \leq a<b$. The length estimated by a local estimator of a parabola $y=\alpha x^{2}, x \in[a, b]$, does not converge when the resolution is rational and tends to zero, to the length of the curve for almost all $\alpha \in\left[0, \frac{1}{2 b}\right]$.

Numerical application: Again, we take the curve $y=g(x)=\frac{1}{\sqrt{2}} x^{2}$ for $x$ between $a=0$ and $b=\frac{1}{\sqrt{2}}$. Suppose that we consider the local estimator Chamfer $5-7-11$ ( 9 ) with $m=2, p(000)=2, p(001)=p(011)=\frac{22}{10}, p(012)=\frac{28}{10}$. With Equation (13), we can prove that the estimation of the length of the parabola given by this local estimator converges to $L_{\text {est }}=\frac{23}{40} \sqrt{2} \approx 0.813172$, this limit is different from the length of the parabola which is $\frac{1}{2}+\frac{1}{4} \sqrt{2} \log (1+\sqrt{2}) \approx 0.811612$. Moreover Figure 4 shows how the length given by the estimator converges to its limit when the resolution tends to zero. It seems on this example that $l(p, g, r)-$ $L_{\text {est }}=O(r)$. 


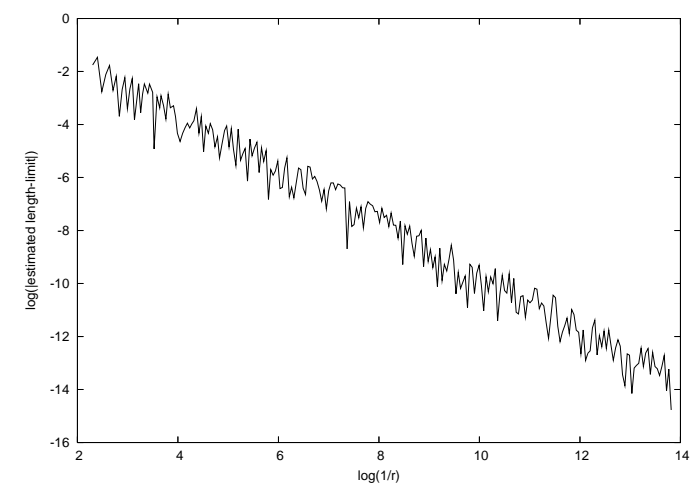

Fig. 4. Figure showing Convergence Speed of the Chamfer 5-7-11 to its limits for the parabola $y=\frac{1}{\sqrt{2}} x^{2}, \quad 0 \leq x \leq \frac{1}{\sqrt{2}}$.

\section{Conclusion and Perspectives}

In this paper, we have proved some local properties of discretizations of parabolas: First we show that locally discretization of parabola and discretization of its tangent often coincide (Tangent Lemma: Lemmas 11 and Theorem 2). In particular, asymptotically, the local patterns of a discretized parabola are digital segments. From this, we also give an explicit formula for the limit of frequency of a pattern of a parabola when the resolution tends to zero (Theorem 1). This has the important consequence that we can know to what tend local estimators of length for the parabolas, moreover it can be proved that this limit is often different from the length of the curve.

This work mainly brings two perspectives:

- The extension of Formula (3), which gives the limit of the frequency of pattern when the resolution tends to zero, to more general curves, in particular to the curves $y=P(x)$ when $P$ is a polynomial of degree greater than 2 .

- The application of this work for recognition of curve by just looking at patterns. For example, if the frequencies of patterns of a curve does not satisfy Theorem 1 then it is not a parabola of equation $y=\alpha x^{2}$.

\section{References}

1. Coeurjolly, D., Klette, R.: A comparative evaluation of length estimators. IEEE Trans. Pattern Anal. Mach. Intell. 26(2) (2004) 252-258

2. Tajine, M., Daurat, A.: On local definitions of length of digital curves. In: Proc. of DGCI 2003. Volume 2886 of Lecture Notes in Comp. Sci. (2003) 114-123

3. Tajine, M.: Digital segments and Hausdorff discretization. In: Proc. of IWCIA 2008. Volume 4958 of Lecture Notes in Comp. Sci. (2008) 75-86

4. Daurat, A., Tajine, M., Zouaoui, M.: About the frequencies of some patterns in digital planes. Application to area estimators. Comput. Graph. 33(1) (2008) 11-20 
5. Granville, A., Rudnick, Z.: Uniform distribution. In Granville, A., Rudnick, Z., eds.: Equidistribution in Number Theory, An Introduction. Springer (2007) 1-13

6. Vinogradov, I.M.: Elements of Number Theory, 5th rev. ed. Dover (1954)

7. Davenport, H.: Multiplicative Number Theory, 2nd ed. Springer-Verlag, New York (1980)

8. Rudin, W.: Real and complex analysis. McGraw-Hill, New York (1966)

9. Borgefors, G.: Distance transformations in digital images. Computer Vision, Graphics, and Image Processing 34(3) (1986) 344-371

10. McIlroy, M.D.: A note on discrete representation of lines. ATT Tech. J. 64(2) (1985) 481-490

11. Wikipedia: (2008) http://en.wikipedia.org/wiki/Pigeonhole_principle accessed 14-November-2008.

12. Munroe, M.E.: Measure and integration, 2nd ed. Addison-Wesley (1971)

\section{A Proofs}

\section{Additional notations}

- The distance between $x$ and $\mathbb{Z}$ is denoted $\|x\|$. So $\|x\|=\min (\langle x\rangle, 1-\langle x\rangle)$.

- If $z$ is complex number $\bar{z}$ denotes its conjugate, and $\operatorname{Re}(z)$ denotes its real part.

$-\operatorname{gcd}(p, q)$ denotes the greatest common divisor of $p$ and $q$.

\section{A.1 Proof of Lemma 3}

For any $f \in L^{1}([0,1])$ we define:

$$
S_{r, k}(f)=\frac{1}{N_{r}} \sum_{X=A_{r}}^{B_{r}} f\left(\left\langle P_{r, k}(X)\right\rangle\right) .
$$

so we have $T_{r, k}(I)=S_{r, k}\left(\chi_{I}\right)$ where $\chi_{I}$ is the indicator function of $I$. We denote $e(t)=e^{2 \pi i t}$ and $e_{c}(t)=e(c t)$.

Sublemma 5 For any $c \in \mathbb{Z} \backslash\{0\}$ we have $S_{r, k}\left(e_{c}\right) \underset{\substack{r \rightarrow 0 \\ r \in \mathbb{Q}}}{\longrightarrow} 0$.

Proof.

$$
\begin{aligned}
\left|S_{r, k}\left(e_{c}\right)\right|^{2} & =\overline{S_{r, k}\left(e_{c}\right)} S_{r, k}\left(e_{c}\right) \\
& =\frac{1}{N_{r}^{2}} \sum_{A_{r} \leq X, Y \leq B_{r}} \overline{e_{c}\left(P_{r, k}(X)\right)} e_{c}\left(P_{r, k}(Y)\right) \\
& =\frac{1}{N_{r}}+\frac{2}{N_{r}^{2}} \operatorname{Re}\left(\sum_{A_{r} \leq X<Y \leq B_{r}} e_{c}\left(P_{r, k}(Y)-P_{r, k}(X)\right)\right)
\end{aligned}
$$


Let us pose $Y=X+h$, we have

$$
\begin{aligned}
P_{r, k}(Y)-P_{r, k}(X) & =r \alpha(X+h)^{2}+2 \alpha k(X+H)-\alpha r X^{2}-2 \alpha k r X \\
& =P_{r, k}(h)+2 \alpha r X h
\end{aligned}
$$

So:

$$
\begin{aligned}
\left|S_{r, k}\left(e_{c}\right)\right|^{2} & =\frac{1}{N_{r}}+\frac{2}{N_{r}^{2}} \operatorname{Re}\left(\sum_{X=A_{r}}^{B_{r}-1} \sum_{h=1}^{B_{r}-X} e_{c}\left(P_{r, k}(h)\right) e_{c}(2 \alpha r X h)\right) \\
& =\frac{1}{N_{r}}+\frac{2}{N_{r}^{2}} \operatorname{Re}\left(\sum_{h=1}^{N_{r}-1} \sum_{X=A_{r}}^{B_{r}-h} e_{c}\left(P_{r, k}(h)\right) e_{c}(2 \alpha r X h)\right) \\
& =\frac{1}{N_{r}}+\frac{2}{N_{r}^{2}} \operatorname{Re}\left(\sum_{h=1}^{N_{r}-1}\left(e_{c}\left(P_{r, k}(h)\right) \sum_{X=A_{r}}^{B_{r}-h} e_{c}(2 \alpha r X h)\right)\right)
\end{aligned}
$$

Subsublemma 6 For any $\beta \in \mathbb{R} \backslash \mathbb{Z}, u, v \in \mathbb{Z}$ such that $u \leq v$ we have:

$$
\left|\sum_{k=u}^{v} e(\beta k)\right| \leq \min \left(v-u+1, \frac{1}{2\|\beta\|}\right) .
$$

Proof.

$$
\sum_{k=u}^{v} e(\beta k)=e(\beta u) \frac{1-e(\beta(v-u+1))}{1-e(\beta)}
$$

so

$$
\left|\sum_{k=u}^{v} e(\beta k)\right| \leq \frac{2}{|1-e(\beta)|}
$$

But $|1-e(\beta)|=\left|e\left(\frac{\beta}{2}\right)\left(e\left(-\frac{\beta}{2}\right)-e\left(\frac{\beta}{2}\right)\right)\right|=2|\sin (\pi \beta)|$. Moreover $\sin (\pi \beta) \geq 2\|\beta\|$ (because $\sin (\pi x) \geq 2 x$ for $\left.x \in\left[0, \frac{1}{2}\right]\right)$. So:

$$
\left|\sum_{k=u}^{v} e(\beta k)\right| \leq \frac{1}{2\|\beta\|}
$$

With the clear inequality $\left|\sum_{k=u}^{v} e(\beta k)\right| \leq v-u+1$, it ends the proof of Subsublemma 6 .

As $\operatorname{Re}(x) \leq|x|$, Subsublemma 6 shows that:

$$
\left|S_{r, k}\left(e_{c}\right)\right|^{2} \leq \frac{1}{N_{r}}+\frac{2}{N_{r}^{2}} \sum_{h=1}^{N_{r}-1} \min \left(N_{r}, \frac{1}{2\|2 \alpha r c h\|}\right)
$$


Subsublemma 7 Let $C_{0} \geq 0, u \in \mathbb{R} \backslash \mathbb{Q}, N, p, q \in \mathbb{N}$ which satisfy $q \leq C_{0} N$, $|u q-p| \leq \frac{1}{C_{0} N}, \operatorname{gcd}(p, q)=1$. We have

$$
\sum_{h=1}^{N-1} \min \left(N, \frac{1}{2\|u h\|}\right) \leq 4\left(1+C_{0}\right)\left(\frac{N^{2}}{q}+N \log q\right) .
$$

Proof.

$$
\sum_{h=1}^{N-1} \min \left(N, \frac{1}{2\|u h\|}\right) \leq \sum_{H=0}^{\left\lfloor\frac{N-1}{q}\right\rfloor} \sum_{h=q H+1}^{q H+q} \min \left(N, \frac{1}{2\|u h\|}\right)
$$

But:

$$
\begin{aligned}
\sum_{h=q H+1}^{q H+q} & \min \left(N, \frac{1}{2\|u h\|}\right)=\sum_{h=1}^{q} \min \left(N, \frac{1}{2\|u(q H+h)\|}\right) \\
\|u(q H+h)\| & =\left\|\left(\frac{p+\varepsilon}{q}\right)(q H+h)\right\| \quad \text { where } \varepsilon=u q-p \\
& =\left\|p H+\frac{p}{q} h+\varepsilon H+\frac{\varepsilon h}{q}\right\| \\
& =\left\|\frac{p}{q} h+\varepsilon H+\frac{\varepsilon h}{q}\right\|
\end{aligned}
$$

Let $\gamma=(\varepsilon H) \bmod \left(\frac{1}{q}\right)$, and $k_{0}$ such that $\varepsilon H=\gamma+\frac{k_{0}}{q}$. We have: $\left(\frac{p}{q} h+\right.$ $\varepsilon H) \bmod 1=\gamma+\frac{i_{h}}{q}$ where $i_{h}=\left(p h+k_{0} q\right) \bmod q . \operatorname{As} \operatorname{gcd}(p, q)=1,\left\{i_{h} \mid h \in\right.$ $\llbracket 1, q \rrbracket\}=\llbracket 0, q-1 \rrbracket$.

If $i_{h}<\frac{q}{2}$ then

$$
\begin{array}{rlrl}
\|u(q H+h)\| & =\left\|\gamma+\frac{i_{h}}{q}+\frac{\varepsilon h}{q}\right\| & \\
& \geq\left\|\gamma+\frac{i_{h}}{q}\right\|-\frac{|\varepsilon| h}{q} \quad \text { because } x \mapsto\|x\| \text { is Lipschitz continuous with constant } 1 \\
& =\gamma+\frac{i_{h}}{q}-\frac{|\varepsilon| h}{q} & \\
& \geq \frac{i_{h}}{q}-|\varepsilon| & \text { as } h \leq q \\
& \geq \frac{i_{h}}{q}-\frac{1}{C_{0} N} .
\end{array}
$$

Similarly if $i_{h} \geq \frac{q}{2}$ then

$$
\begin{aligned}
\|u(q H+h)\| & \geq\left\|\gamma+\frac{i_{h}}{q}\right\|-\frac{|\varepsilon| h}{q} \\
& =1-\left(\gamma+\frac{i_{h}}{q}\right)-\frac{|\varepsilon| h}{q} \\
& \geq \frac{q-1-i_{h}}{q}-\frac{1}{C_{0} N} .
\end{aligned}
$$


If we use the variable change $h \mapsto i_{h}$ for $i_{h}<\frac{q}{2}, h \mapsto q-1-i_{h}$ for $i_{h} \geq \frac{q}{2}$, the inequality $\min \left(N, \frac{1}{2\|u(q H+h)\|}\right) \leq N$ for $i_{h}=0,1, q-2, q-1$, and the inequalities (8), (8) for the other $i_{h}$, we deduce:

$$
\sum_{h=1}^{q} \min \left(N, \frac{1}{2\|u(q H+h)\|}\right) \leq 4 N+2 \sum_{i=2}^{\left\lfloor\frac{q-1}{2}\right\rfloor} \frac{1}{2\left(\frac{i}{q}-\frac{1}{C_{0} N}\right)}
$$

But $\frac{1}{1-x} \leq 1+2 x$ for $x \in\left(0, \frac{1}{2}\right]$. So for $i \geq 2$ we have:

$$
\begin{aligned}
\frac{1}{\frac{i}{q}-\frac{1}{C_{0} N}} & =\frac{q}{i} \frac{1}{1-\frac{q}{i C_{0} N}} \\
& \leq \frac{q}{i}\left(1+2 \frac{q}{i C_{0} N}\right) \quad \text { because } \frac{q}{i C_{0} N} \in\left(0, \frac{1}{2}\right] \text { as } i \geq 2 \text { and } q \leq C_{0} N \\
& \leq 2 \frac{q}{i}
\end{aligned}
$$

So

$$
\sum_{h=1}^{q} \min \left(N, \frac{1}{2\|u(q H+h)\|}\right) \leq 4 N+2 q \sum_{i=2}^{\left\lfloor\frac{q-1}{2}\right\rfloor} \frac{1}{i}
$$

But:

$$
\begin{aligned}
\sum_{i=2}^{\left\lfloor\frac{q-1}{2}\right\rfloor} \frac{1}{i} & =\sum_{i=2}^{\left\lfloor\frac{q-1}{2}\right\rfloor} \int_{i-1}^{i} \frac{1}{i} \mathrm{~d} t \\
& \leq \sum_{i=2}^{\left\lfloor\frac{q-1}{2}\right\rfloor} \int_{i-1}^{i} \frac{1}{t} \mathrm{~d} t \\
& =\int_{1}^{\left\lfloor\frac{q-1}{2}\right\rfloor} \frac{1}{t} \mathrm{~d} t \\
& =\log \left(\left\lfloor\frac{q-1}{2}\right\rfloor\right)
\end{aligned}
$$

So:

$$
\begin{array}{rlrl}
\sum_{h=1}^{N-1} \min \left(N, \frac{1}{2\|u h\|}\right) & \leq\left(\left\lfloor\frac{N-1}{q}\right\rfloor+1\right)\left(4 N+2 q \log \left(\left\lfloor\frac{q-1}{2}\right\rfloor\right)\right) \\
& \leq\left(\frac{N}{q}+1\right)(4 N+2 q \log q) & \\
& \leq\left(\frac{N}{q}+\frac{C_{0} N}{q}\right)(4 N+2 q \log q) & \text { because } q \leq C_{0} N \\
& \leq 4\left(1+C_{0}\right)\left(N^{2}+N \log q\right)
\end{array}
$$

This finishes the proof of Subsublemma 7 . 
In the following we suppose that $r$ is rational. So $\alpha r$ is irrational, so by Dirichlet's principle (11]) we know that there exist two coprime integers $q_{r} \leq \frac{2 N_{r}}{\alpha c(b-a)}$ and $p_{r}$ such that $\left|2 \alpha r c q_{r}-p_{r}\right| \leq \frac{\alpha c(b-a)}{2 N_{r}}$. By Subsublemma 团 with $C_{0}=\frac{2}{\alpha c(b-a)}$ and Equation (5) we deduce:

$$
\begin{aligned}
\left|S_{r, k}\left(e_{c}\right)\right|^{2} & \leq \frac{1}{N_{r}}+\frac{8\left(1+C_{0}\right)}{N_{r}^{2}}\left(\frac{N_{r}^{2}}{q}+N_{r} \log q_{r}\right) \\
& \leq \frac{1}{N_{r}}+\frac{8\left(1+C_{0}\right)}{q_{r}}+\frac{8\left(1+C_{0}\right) \log q_{r}}{N_{r}}
\end{aligned}
$$

As $N_{r} \underset{r \rightarrow 0}{\longrightarrow}+\infty$ and $\frac{\log q_{r}}{N_{r}} \leq \frac{\log \left(C_{0} N_{r}\right)}{N_{r}} \underset{r \rightarrow 0}{\longrightarrow} 0$, it remains to prove that $q_{r} \underset{r \rightarrow 0}{\longrightarrow}$ $+\infty$.

We have:

$$
\frac{p_{r}}{q_{r}} \geq 2 \alpha r c-\frac{\alpha c(b-a)}{2 N_{r}}
$$

We have $N_{r} \geq\left(\frac{b}{r}-1\right)-\left(\frac{a}{r}+1\right)-1=\frac{b-a-r}{r}$. We suppose without loss of generality that $r \leq \frac{b-a}{2}$ so $N_{r} \geq \frac{b-a}{2 r}$, so

$$
\frac{p_{r}}{q_{r}} \geq 2 \alpha r c-\alpha r c>0
$$

so $p_{r} \geq 1$. We deduce:

$$
\frac{1}{q_{r}} \leq \frac{p_{r}}{q_{r}} \leq 2 \alpha r c+\frac{1}{N_{r} q_{r}} \leq 2 \alpha r c+\frac{1}{N_{r}}
$$

So $q_{r} \geq \frac{1}{2 \alpha r c+\frac{1}{N_{r}}} \underset{r \rightarrow 0}{\longrightarrow}+\infty$. This ends the proof of Sublemma 5.

To finish the proof of Lemma 3 we must prove that $S_{r, k}\left(\chi_{I}\right) \underset{\substack{r \rightarrow 0 \\ r \in \mathbb{Q}}}{\longrightarrow} \mu(I)$ where $\chi_{I}$ is the indicator function of $I$. We have $S_{r, k}\left(e_{0}\right)=1$ and by Sublemma 5 $S_{r, k}\left(e_{c}\right) \underset{\substack{r \rightarrow 0 \\ r \in \mathbb{Q}}}{\longrightarrow} 0$ for $c \neq 0$, so by Weyl's strategy (see for example [2] or 4 , Appendix A.1]) we can prove Lemma 3 .

\section{A.2 Proof of Theorem 1}

Now let:

$$
G_{r}^{g}(\omega)=\frac{\operatorname{card}\left\{X \in \llbracket A_{r}, B_{r}-m \rrbracket \mid s_{m}^{g^{\prime}(r X),\left\langle\frac{g(r X)}{r}\right\rangle}=\omega\right\}}{N_{r}-m} .
$$

By Lemma 11 we have

$$
F_{r}^{g}(\omega)-G_{r}^{g}(\omega) \underset{\substack{r \rightarrow 0 \\ r \in \mathbb{Q}}}{\longrightarrow} 0 .
$$


We have by definition of $P I(\omega)$ (and because $\left\lfloor\left\langle\frac{g(r X)}{r}\right\rangle\right\rfloor=0$ ):

$$
G_{r}^{g}(\omega)=\frac{\operatorname{card}\left\{X \in \llbracket A_{r}, B_{r}-m \rrbracket \mid\left(g^{\prime}(r X),\left\langle\frac{g(r X)}{r}\right\rangle\right) \in P I(\omega)\right\}}{N_{r}-m}
$$

For any subset $E$ of $\mathbb{R}^{2}$, we define:

$$
H_{r}(E)=\frac{\operatorname{card}\left\{X \in \llbracket A_{r}, B_{r} \rrbracket \mid\left(g^{\prime}(r X),\left\langle\frac{g(r X)}{r}\right\rangle\right) \in E\right\}}{N_{r}}
$$

We have

$$
\frac{N_{r} H_{r}(P I(\omega))-m}{N_{r}-m} \leq G_{r}^{g}(\omega) \leq \frac{N_{r} H_{r}(P I(\omega))}{N_{r}-m} .
$$

Suppose first that $E=\left[\alpha_{1}, \alpha_{2}\right) \times I$. The function $g^{\prime}$ is linear, so in particular is a bijection, we denote by $g^{\prime-1}$ its reciprocal function. We suppose that $\alpha>$ 0 , so that $g^{\prime}$ is an increasing function. $\left(g^{\prime}(r X),\left\langle\frac{g(r X)}{r}\right\rangle\right) \in E$ is equivalent to $g^{\prime}(r X) \in\left[\alpha_{1}, \alpha_{2}\right)$ and $\left.\left\langle\frac{g(r X)}{r}\right\rangle\right) \in I$. So

$$
H_{r}(E)=\frac{\operatorname{card}\left\{X \in \llbracket\left\lceil\frac{g^{\prime-1}\left(\alpha_{1}\right)}{r}\right\rceil,\left\lfloor\frac{g^{\prime-1}\left(\alpha_{2}\right)}{r}\right\rfloor \rrbracket \mid\left\langle\frac{g(r X)}{r}\right\rangle \in I\right\}}{N_{r}} .
$$

If we apply Lemma 3 with $k=0$ to the piece of the curve $y=g(x)$ restricted to the domain $g^{\prime-1}\left(\alpha_{1}\right) \leq x \leq g^{\prime-1}\left(\alpha_{2}\right)$. we find:

$$
\frac{N_{r} H_{r}(E)}{\left\lfloor\frac{g^{\prime-1}\left(\alpha_{2}\right)}{r}\right\rfloor-\left\lceil\frac{g^{\prime-1}\left(\alpha_{1}\right)}{r}\right\rceil+1} \underset{\substack{r \rightarrow 0 \\ r \in \mathbb{Q}}}{\longrightarrow} \mu(I)
$$

We deduce that:

$$
H_{r}\left(\left[\alpha_{1}, \alpha_{2}\right) \times I\right) \underset{\substack{r \rightarrow 0 \\ r \in \mathbb{Q}}}{\longrightarrow} \frac{g^{-1}\left(\alpha_{2}\right)-g^{-1}\left(\alpha_{1}\right)}{b-a} \mu(I)
$$

In [10] it is shown that

$$
P I(\omega)=\left\{(\alpha, \beta) \mid \alpha \in\left[\alpha_{b}, \alpha_{e}\right] \text { and } \operatorname{pinf}_{\alpha}(\omega) \leq \beta<\operatorname{psup}_{\alpha}(\omega)\right.
$$

where $\alpha \mapsto \operatorname{pinf}_{\alpha}(\omega)$ and $\alpha \mapsto \operatorname{psup}_{\alpha}(\omega)$ are two piecewise affine functions which slope is between $-m$ and 0 . 1 .

Let $n \in \mathbb{N} \backslash\{0\}$ and $y_{i}=\alpha_{b}+i \frac{\alpha_{e}-\alpha_{b}}{n}$ for $i \in \llbracket 0, n \rrbracket$.

$P I(\omega)$ is approximated by an union of $n$ rectangles:

$$
\bigcup_{i=1}^{n}\left[y_{i-1}, y_{i}\right) \times\left[\operatorname{pinf}_{y_{i-1}}(\omega), \operatorname{psup}_{y_{i}}(\omega)\right) \subset P I(\omega) \subset \bigcup_{i=1}^{n}\left[y_{i-1}, y_{i}\right] \times\left[\operatorname{pinf}_{y_{i}}(\omega), \operatorname{psup}_{y_{i-1}}(\omega)\right)
$$

\footnotetext{
${ }^{1}$ In fact it is proved that $P I(\omega)$ is a triangle or a quadrangle.

2 Formulas (11), (2) do not define pinf and psup for $\alpha=\alpha_{b}, \alpha_{e}$, we define these values just by continuity.
} 
As $y_{i}-y_{i-1} \leq \frac{1}{n}$ and $\alpha \mapsto \operatorname{pinf}_{\alpha}(\omega)$ and $\alpha \mapsto \operatorname{psup}_{\alpha}(\omega)$ are two piecewise affine functions which slope is between $-m$ and 0 , we have $\operatorname{pinf}_{y_{i-1}}(\omega) \leq \operatorname{pinf}_{y_{i}}(\omega)+\frac{m}{n}$ and $\operatorname{psup}_{y_{i-1}}(\omega) \leq \operatorname{psup}_{y_{i}}(\omega)+\frac{m}{n}$. So:

$$
\begin{aligned}
& \bigcup_{i=1}^{n}\left[y_{i-1}, y_{i}\right) \times\left[\operatorname{pinf}_{y_{i}}(\omega)+\frac{m}{n}, \operatorname{psup}_{y_{i}}(\omega)\right) \subset P I(\omega) \subset \\
& \bigcup_{i=1}^{n}\left[y_{i-1}, y_{i}\right] \times\left[\operatorname{pinf}_{y_{i}}(\omega), \operatorname{psup}_{y_{i}}(\omega)+\frac{m}{n}\right)
\end{aligned}
$$

Let

$$
\begin{aligned}
& F_{n, r}=H_{r}\left(\bigcup_{i=1}^{n}\left[y_{i-1}, y_{i}\right) \times\left[\operatorname{pinf}_{y_{i}}(\omega)+\frac{m}{n}, \operatorname{psup}_{y_{i}}(\omega)\right)\right), \\
& F_{n, r}^{\prime}=H_{r}\left(\bigcup_{i=1}^{n}\left[y_{i-1}, y_{i}\right] \times\left[\operatorname{pinf}_{y_{i}}(\omega), \operatorname{psup}_{y_{i}}(\omega)+\frac{m}{n}\right)\right) .
\end{aligned}
$$

So by Equation (12):

$$
F_{n, r} \leq H_{r}(P I(\omega)) \leq F_{n, r}^{\prime} .
$$

By summing equations of the form (11) we obtain:

$$
\begin{aligned}
& F_{n, r} \underset{\substack{r \rightarrow 0 \\
r \in \mathbb{Q}}}{\longrightarrow} \sum_{i=1}^{n} \frac{g^{-1}\left(y_{i}\right)-g^{-1}\left(y_{i-1}\right)}{b-a}\left(F L_{y_{i}}(\omega)-\frac{m}{n}\right) \\
& F_{n, r}^{\prime} \underset{\substack{r \rightarrow 0 \\
r \in \mathbb{Q}}}{\longrightarrow} \sum_{i=1}^{n} \frac{g^{\prime-1}\left(y_{i}\right)-g^{\prime-1}\left(y_{i-1}\right)}{b-a}\left(F L_{y_{i}}(\omega)+\frac{m}{n}\right)
\end{aligned}
$$

Let

$$
\begin{gathered}
F_{n}^{\prime \prime}=\sum_{i=1}^{n} \frac{g^{\prime-1}\left(y_{i}\right)-g^{-1}\left(y_{i-1}\right)}{b-a} F L_{y_{i}}(\omega) . \\
\left.\lim _{\substack{r \rightarrow 0 \\
r \in \mathbb{Q}}} F_{n, r}\right)-F_{n}^{\prime \prime}=-\sum_{i=1}^{n} \frac{g^{\prime-1}\left(y_{i}\right)-g^{\prime-1}\left(y_{i-1}\right)}{b-a} \frac{m}{n} \\
=-\sum_{i=1}^{n} \frac{\frac{1}{2 \alpha}\left(y_{i}-y_{i-1}\right)}{b-a} \frac{m}{n} \\
=-\frac{m\left(\alpha_{e}-\alpha_{b}\right)}{2 \alpha(b-a)} \frac{1}{n}
\end{gathered}
$$

Similarly

$$
\left(\lim _{\substack{r \rightarrow 0 \\ r \in \mathbb{Q}}} F_{n, r}^{\prime}\right)-F_{n}^{\prime \prime}=\frac{m\left(\alpha_{e}-\alpha_{b}\right)}{2 \alpha(b-a)} \frac{1}{n} .
$$


Let $z_{i}=g^{\prime-1}\left(y_{i}\right)$, we have

$$
F_{n}^{\prime \prime}=\sum_{i=1}^{n}\left(z_{i}-z_{i-1}\right) \frac{F L_{g^{\prime}\left(z_{i}\right)}(\omega)}{b-a}
$$

which is a Riemann sum of $x \mapsto \frac{F L_{g^{\prime}(x)}(\omega)}{b-a}$, but $t \mapsto F L_{t}(\omega)$ is continuous ([3]), so we have by [12, Chap. 5]:

$$
F_{n}^{\prime \prime} \underset{n \rightarrow \infty}{\longrightarrow} \frac{1}{b-a} \int_{a}^{b} F L_{g^{\prime}(x)}(\omega) \mathrm{d} x
$$

Let $\varepsilon>0$, there exists $N_{1}$ such that for all $n>N_{1}$ we have

$$
\left|F_{n}^{\prime \prime}-\frac{1}{b-a} \int_{a}^{b} F L_{g^{\prime}(x)}(\omega) \mathrm{d} x\right| \leq \frac{\varepsilon}{3} .
$$

There exists $N_{2}$ such that for any $n>N_{2}$ we have

$$
\frac{m\left(\alpha_{e}-\alpha_{b}\right)}{2 \alpha(b-a)} \frac{1}{n}<\frac{\varepsilon}{3}
$$

Let $N=\max \left(N_{1}, N_{2}\right)+1$. There exists $R_{1}>0$ such that for any rational $r<R_{1}$ we have:

$$
\left|F_{N, r}-\left(F_{N}^{\prime \prime}-\frac{m\left(\alpha_{e}-\alpha_{b}\right)}{2 \alpha(b-a)} \frac{1}{N}\right)\right| \leq \frac{\varepsilon}{3} .
$$

There exists $R_{2}>0$ such that for any rational $r<R_{2}$ we have:

$$
\left|F_{N, r}^{\prime}-\left(F_{N}^{\prime \prime}+\frac{m\left(\alpha_{e}-\alpha_{b}\right)}{2 \alpha(b-a)} \frac{1}{N}\right)\right| \leq \frac{\varepsilon}{3} .
$$

Suppose that $r<\min \left(R_{1}, R_{2}\right)$.

We have:

$$
\begin{aligned}
H_{r}(P I(\omega)) & \geq F_{n, r} \\
& \geq F_{N}^{\prime \prime}-\frac{m\left(\alpha_{e}-\alpha_{b}\right)}{2 \alpha(b-a)} \frac{1}{N}-\frac{\varepsilon}{3} \\
& \geq F_{N}^{\prime \prime}-\frac{\varepsilon}{3}-\frac{\varepsilon}{3} \\
& \geq \frac{1}{b-a} \int_{a}^{b} F L_{g^{\prime}(x)}(\omega) \mathrm{d} x-\frac{\varepsilon}{3}-\frac{\varepsilon}{3}-\frac{\varepsilon}{3} \\
& =\frac{1}{b-a} \int_{a}^{b} F L_{g^{\prime}(x)}(\omega) \mathrm{d} x-\varepsilon
\end{aligned}
$$

Similarly

$$
\begin{aligned}
H_{r}(P I(\omega)) & \leq F_{n, r}^{\prime} \\
& \leq \frac{1}{b-a} \int_{a}^{b} F L_{g^{\prime}(x)}(\omega) \mathrm{d} x+\varepsilon
\end{aligned}
$$


SO

$$
H_{r}(P I(\omega)) \underset{\substack{r \rightarrow 0 \\ r \in \mathbb{Q}}}{\longrightarrow} \frac{1}{b-a} \int_{a}^{b} F L_{g^{\prime}(x)}(\omega) \mathrm{d} x
$$

With (9) and (10) this proves Theorem 1 .

\section{A.3 Proof of Theorem 3}

It is easy to see that:

$$
l(p, g, r)-\frac{(b-a)}{m} \sum_{\omega \in \mathcal{P}_{m}} p(\omega) F_{r}^{\prime g}(\omega) \underset{r \rightarrow 0}{\longrightarrow} 0
$$

where

$$
F_{r}^{\prime g}=\frac{\operatorname{card}\left\{X \in \llbracket A_{r}, B_{r}-m \rrbracket \cap\left(A_{r}+m \mathbb{Z}\right) \mid \omega_{X, r, m}^{g}=\omega\right\}}{\left\lfloor\frac{B_{r}-m-A_{r}}{m}\right\rfloor} .
$$

Consider again the curve defined by $g(x)=\alpha x^{2}$ for $\alpha \notin \mathbb{Q}$. The proof of previous section can be extended to prove:

$$
F_{r}^{\prime g} \underset{r \in \mathbb{Q}}{\longrightarrow} \frac{1}{b-a} \int_{a}^{b} F L_{g^{\prime}(x)}(\omega) \mathrm{d} x
$$

so

$$
l(p, g, r) \underset{\substack{r \rightarrow 0 \\ r \in \mathbb{Q}}}{\longrightarrow} \frac{1}{m} \sum_{\omega \in \mathcal{P}_{m}} p(\omega) \int_{a}^{b} F L_{g^{\prime}(x)}(\omega) \mathrm{d} x .
$$

Let $F_{n}$ the $n$-th Farey of order $m$.

We know that $x \mapsto F L_{x}(\omega)$ is affine on the intervals $\left[F_{i}, F_{i+1}\right]$ ([3]). So for each $i$ there exists $u_{i}, v_{i}$ we have $F L_{x}(\omega)=u_{i} x+v_{i}$.

Let $k_{\alpha}$ the smallest integer $k$ such that $2 \alpha a \leq F_{k}$ and $l_{\alpha}$ the biggest integer $l$ such that $F_{l} \leq 2 \alpha b$. We deduce that

$$
\begin{aligned}
\int_{a}^{b} F L_{g^{\prime}(x)}(\omega) \mathrm{d} x & =\int_{a}^{\frac{F_{k_{\alpha}}}{2 \alpha}}\left(u_{k_{\alpha}-1}(2 \alpha x)+v_{k_{\alpha}-1}\right) \mathrm{d} x+\sum_{i=k_{\alpha}}^{l_{\alpha}-1} \int_{\frac{F_{i}}{2 \alpha}}^{\frac{F_{i+1}}{2 \alpha}}\left(u_{i}(2 \alpha x)+v_{i}\right) \mathrm{d} x+ \\
\int_{\frac{F_{l_{\alpha}}}{2 \alpha}}^{b}\left(u_{l_{\alpha}}(2 \alpha x)+v_{l \alpha}\right) \mathrm{d} x & \\
& =\left[u_{k_{\alpha}-1} \alpha x^{2}+v_{k_{\alpha}-1} x\right]_{a}^{\frac{F_{k_{\alpha}}}{2 \alpha}}+\sum_{i=k_{\alpha}}^{l_{\alpha}-1}\left[u_{i} \alpha x^{2}+v_{i} x\right]_{\frac{F_{i}}{2 \alpha}}^{\frac{F_{i+1}}{2 \alpha}}+\left[u_{l_{\alpha}} \alpha x^{2}+v_{l_{\alpha}} x\right]_{\frac{F_{l_{\alpha}}}{2 \alpha}}^{b} \\
& =\frac{A_{\alpha, \omega}}{\alpha}+B_{\alpha, \omega} \alpha+C_{\alpha, \omega}
\end{aligned}
$$


where

$$
\begin{aligned}
A_{\alpha, \omega} & =\frac{1}{4}\left(u_{k_{\alpha}-1} F_{k_{\alpha}}^{2}+2 v_{k_{\alpha}-1} F_{k_{\alpha}}+\sum_{i=k_{\alpha}}^{l_{\alpha}-1}\left[u_{i} x^{2}+2 v_{i} x\right]_{F_{i}}^{F_{i+1}}-u_{l_{\alpha}} F_{l_{\alpha}}^{2}-2 v_{l_{\alpha}} F_{l_{\alpha}}\right) \\
B_{\alpha, \omega} & =u_{l_{\alpha}} b^{2}-u_{k_{\alpha}-1} a^{2} \\
C_{\alpha, \omega} & =v_{l_{\alpha}} b-v_{k_{\alpha}-1} a
\end{aligned}
$$

As $k_{\alpha}$ and $l_{\alpha}$ are constant on the intervals which contains no reals $\frac{F_{i}}{2 a}$ and $\frac{F_{i}}{2 b}$, we can partition the interval $\left[0, \frac{1}{2 b}\right]$ in a finite number of intervals $\left(I_{k}\right)_{0 \leq k \leq n}$ such that the functions $\alpha \mapsto A_{\alpha, \omega}, B_{\alpha, \omega}, C_{\alpha, \omega}$ are constant on each interval $I_{k}$.

By (5), we have:

$$
\lim _{\substack{r \rightarrow 0 \\ r \in \mathbb{Q}}} l(p, g, r)=\frac{A_{\alpha}}{\alpha}+B_{\alpha} \alpha+C_{\alpha}
$$

where

$$
A_{\alpha}=\frac{1}{m} \sum_{\omega \in \mathcal{P}_{m}} p(\omega) A_{\alpha, \omega}, \quad B_{\alpha}=\frac{1}{m} \sum_{\omega \in \mathcal{P}_{m}} p(\omega) B_{\alpha, \omega}, \quad C_{\alpha}=\frac{1}{m} \sum_{\omega \in \mathcal{P}_{m}} p(\omega) C_{\alpha, \omega}
$$

So $L_{e s t}(\alpha)=\lim _{\substack{r \in \mathbb{Q} \\ r \in \mathbb{Q}}} l(p, g, r)$ is of the form $\frac{A}{\alpha}+B \alpha+C$ on each interval $I_{k}$.

Let $L_{\text {real }}(\alpha)$ the true length of the parabola curve $\left\{\left(x, \alpha x^{2}\right) \mid x \in[a, b]\right\}$. We have:

$$
\begin{aligned}
L_{\text {real }}(\alpha) & =\int_{a}^{b} \sqrt{1+(2 \alpha x)^{2}} \mathrm{~d} x \\
& =\left[\frac{x \sqrt{1+(2 \alpha x)^{2}}}{2}+\frac{\arg \sinh (2 \alpha x)}{4 \alpha}\right]_{a}^{b}
\end{aligned}
$$

Suppose that $L_{\text {real }}(\alpha)=L_{\text {est }}(\alpha)$ for an infinite number of irrational $\alpha$ then there exists an interval $I_{k}$ of the previous partition of $\left[0, \frac{1}{2 b}\right]$ such that $L_{\text {real }}(\alpha)=$ $L_{e s t}(\alpha)$ for an infinite number of irrational $\alpha \in I_{k}$. On $I_{k}$ we know that $L_{e s t}(\alpha)$ has the form $\frac{A}{\alpha}+B \alpha+C$. The functions $\alpha \mapsto \alpha L_{\text {real }}(\alpha)$ and $\alpha \mapsto \alpha\left(\frac{A}{\alpha}+B \alpha+C\right)$ are holomorphic in a open set of $\mathbb{C}$ containing $\left[0, \frac{1}{2 b}\right]$ and are equal for an infinite number of $\alpha \in I_{k} \subset\left[0, \frac{1}{2 b}\right]$ so by Theorem on the zeros of holomorphic functions [6, Cha. 10] they are equal on $\left[0, \frac{1}{2 b}\right]$. So:

$$
\alpha L_{\text {real }}(\alpha)=A+B \alpha^{2}+C \alpha \quad \text { for all } \alpha \in\left[0, \frac{1}{2 b}\right]
$$

We have:

$$
\begin{aligned}
\frac{\partial\left(\alpha L_{\text {real }}(\alpha)\right.}{\partial \alpha} & =b \sqrt{1+(2 \alpha b)^{2}}-a \sqrt{1+(2 \alpha a)^{2}} \\
& =b-a+2\left(b^{3}-a^{3}\right) \alpha^{2}+o\left(\alpha^{2}\right) \quad \text { when } \alpha \rightarrow 0
\end{aligned}
$$


But $\frac{\partial\left(A+B \alpha^{2}+C \alpha\right)}{\partial \alpha}=2 B \alpha+C$, so $2\left(b^{3}-a^{3}\right)=0$ which is impossible if $b>a$. So the hypothesis that $L_{\text {real }}(\alpha)=L_{\text {est }}(\alpha)$ for an infinite number of irrational $\alpha$ is absurd. 